\title{
Tuberculous meningitis diagnosis and treatment in adults: A series of 189 suspected cases
}

\author{
MENGCHUAN LUO ${ }^{1}$, WEIFEI WANG ${ }^{2}$, QIUMING ZENG ${ }^{1}$, YUEBEI LUO $^{1}$, HUAN YANG $^{1}$ and XIAOSU YANG $^{1}$ \\ ${ }^{1}$ Department of Neurology, Xiangya Hospital, Central South University, Changsha, Hunan 410008; \\ ${ }^{2}$ Department of Neurology, People's Hospital of Liaocheng, Liaocheng, Shandong 252000, P.R. China
}

Received September 16, 2016; Accepted June 29, 2017

DOI: $10.3892 /$ etm.2018.6496

\begin{abstract}
The present study aimed to summarize the clinical features, alterations in cerebrospinal fluid (CSF), imaging characteristics, diagnostic methods, treatment regimens and outcomes of adult Chinese patients with tuberculous meningitis (TBM). Clinical data of 189 cases with 4 cases confirmed with definite TBM, 65 cases of probable (diagnostic score, $\geq 12$ with imaging or $\geq 10$ without imaging) and 120 cases of possible (diagnostic score, 6-11 with imaging or 6-9 without imaging) TBM admitted to Xiangya Hospital of Central South University between January 2009 and January 2015 were investigated retrospectively. Data on the clinical, laboratory and demographic characteristics of patients, as well as the results of radiological investigations and the clinical outcome, were collected for all patients. A total of $89.9 \%$ patients illustrated symptoms of acute or sub-acute TBM. The most frequent symptoms and signs were fever $(78.3 \%)$, headache $(89.2 \%)$, decreased level of consciousness (48.1\%), meningeal irritation (73\%), impairment of cranial nerve function and increased intracranial pressure $(60 \%)$. The CSF protein concentration was significantly elevated and CSF glucose was greatly decreased in these cases. Imaging data were available for 144 cases, with 66 cases presenting abnormal chest X-ray or computed tomography findings, and 127 cases presenting abnormal brain magnetic resonance imaging findings among the 144 patients examined. All patients received anti-tuberculosis (TB) therapy, while 7 patients underwent neurosurgical drainage due to hydrocephalus and 3 patients succumbed to the disease. Among the survivors, $87 \%$ presented significant improvement. In conclusion, the diagnosis of TBM should combine clinical manifestations, CSF examination and the effect of anti-TB therapy. Differential diagnosis and trial anti-TB therapy may be of help for diagnosis. Positive CSF smear, CSF culture and
\end{abstract}

Correspondence to: Dr Xiaosu Yang, Department of Neurology, Xiangya Hospital, Central South University, 87 Xiangya Road, Changsha, Hunan 410008, P.R. China

E-mail: zhongliuke001@yeah.net

Key words: tuberculous meningitis, clinical feature, cerebrospinal fluid, treatment, review biopsy of the brain, or biopsy of meninges are golden standards for the diagnosis of TBM. Early diagnosis and treatment are very important for improving the outcome.

\section{Introduction}

Tuberculous meningitis (TBM) is the most severe type of extrapulmonary tuberculosis (TB), although it is the least common type (incidence, 5-15\%). Approximately $1 / 3$ of the global population is infected with latent TB (1). TBM is associated with a high mortality and morbidity, particularly in developing countries, such as in China, where TBM has the second highest incidence in the world (2). In 2012, there were $~ 8.6$ million incidences of TB globally, and among these, 1.3 million fatalities occurred (3). The diagnosis of TBM remains challenging due to the nonspecific clinical presentation of patients. The initial differential diagnosis includes other bacterial, viral or fungal infections of the central nervous system (CNS), noninfectious inflammatory diseases of the meninges (including systemic lupus erythematosus) and intracranial malignancy (2). The TBM-induced inflammatory reaction is associated with several complications, including cerebrovascular disease, cranial nerve palsy, hydrocephalus and infarction (4). Rapid diagnosis and therapy are therefore necessary to decrease the high-mortality and severe sequelae associated with the disease (4). Due to common characteristics with other diseases and nonspecific clinical presentation, misdiagnosis or delayed diagnosis of TBM results in increased mortality rate (2).

The risk factors of the TBM, including dystrophia, alcoholism, diabetes mellitus and cell-mediated immune mechanism defects, commonly exist in the patients with human immunodeficiency virus (HIV) disease, immunosuppressive diseases, and even in immunocompetent individuals (5). However, alternative factors, including corticosteroid medication, diabetes mellitus and chronic hepatitis and cirrhosis have been associated with the occurrence of TBM in the clinic (6). The standard anti-TBM drugs were applied at all centers, however, the total length of treatment ranged from 6 to 12 months. The World Health Organization suggests treatment between 9-12 months is sufficient for successfully treating TBM (7). The adjuvant prednisolone therapy method has been applied to a large number of TBM patients, and significantly decreased the mortality rates of TBM (8). 
In the present study, 189 patients with confirmed or presumed TBM reported over a 6-year period were analyzed retrospectively. The aims of the current study were to assess the clinical, laboratory and neuroradiological findings of TBM patients and to evaluate the prognostic significance of these variables. In addition, the local epidemiological data were reported from these cases.

\section{Materials and methods}

Patients. The cases included in the present study were patients $>14$ years of age (age range, 15-79 years) with confirmed or highly suspected TBM, who were admitted to the Xiangya Hospital of Central South University (Changsha, China) during the 6-year period between January 2009 and January 2015. Cases with pulmonary TB were excluded. Among 213 patients with confirmed or presumed TBM, 24 were excluded due to insufficient follow-up or incomplete data (5 cases refused lumbar puncture and imaging examination). The clinical, laboratory and radiological characteristics of 189 patients obtained through the retrospective review of hospital and outpatient follow-up reports were evaluated. All of the patients provided their prior informed written consent for the present study.

Diagnosis. The common basic diagnosis of TBM is based on the typical clinical presentation, neuroimaging characteristics, cerebrospinal fluid (CSF) examination, and comprehensive judgment of the response to anti-TB drug treatment (2). A clear diagnosis is based on the discovery of Mycobacterium tuberculosis in CSF cell smears or bacterial culture.

The cases reviewed in the current study were revised based on the standardized clinical case definition that was reported by Bartzatt (2). Based on the aforementioned study, the classification of categories was re-structured as shown in Fig. 1. All patients were classified into the definite, probable, possible and not TBM diagnosis categories according to Marais et al (9). Briefly, in patients meeting the criteria for suspected TBM, lumbar puncture or brain imaging was conducted.

As subsequent examination results became available, patients moved up or down the diagnostic pyramid (Fig. 1), and were finally classified as definite, probable, possible or not TBM according to the diagnostic criteria. Definite TBM is defined as the detection of CNS Mycobacterium tuberculosis infection based on microbiological identification or evidence from commercial nucleic acid amplification tests. For probable TBM diagnosis, a diagnostic score of $\geq 12$ is required when imaging is available, or a score of $\geq 10$ when imaging is not available. Possible TBM is diagnosed in patients with a diagnostic score of 6-11 when imaging is available, while a score of 6-9 is required when imaging is not available.

Clinical stage. According to the classification of the UK Medical Research Council (1), the TBM cases were divided into the following three categories: Stage I, non-specific symptoms and signs, unconscious or vague consciousness, and no damage to the function of the nervous system; stage II, meningeal irritation, mild damage to the function of the nervous system (such as cranial nerve palsy) and motor dysfunction; stage III, seizures or convulsions, drowsiness or coma, and severe neurological dysfunction (including paresis or paralysis).

Other examinations. Patients were also subjected to a lumbar puncture, CSF cytology detection examinations as described previously $(2,9,10)$.

Statistical analysis. Continuous variables are expressed as the mean \pm standard deviation, and categorical variables are presented as a proportion of the total number of patients. Univariate analysis was conducted using $\chi^{2}$ test or Fisher's exact test for categorical variables, and Student's t-test or Mann-Whitney U test for continuous variables, as indicated. All statistical analyses were performed using GraphPad Prism version 5.0 (GraphPad Software, Inc., San Diego, CA, USA). Results were expressed as adjusted odds ratios with the corresponding 95\% confidence interval (CI). A P-value of $<0.05$ was regarded to indicate a statistically significant difference.

\section{Results}

Clinical diagnosis. The imaging data were available for 144 cases, with 66 cases presenting abnormal chest X-ray or computed tomography findings, and 127 cases presenting abnormal brain magnetic resonance imaging findings among the 144 patients examined.

In the present study, 4 cases were confirmed with definite TBM. Probable TBM was diagnosed in 65 cases, including 47 cases where imaging was available (diagnostic score, $\geq 12$ ) and 18 cases where imaging was not available (diagnostic score, $\geq 10$ ). A total of 120 patients were classified as possible TBM cases, including 77 cases where imaging was available (diagnostic score, 6-11) and in 43 cases where imaging was not available (diagnostic score, 6-9).

Clinical data. A total of 189 cases of definite, probable or possible TBM were included in the current study, with an age distribution range of 15-79 years and an average age of $38.38 \pm 17.90$ years (95\% CI, 32.41-44.35 years). As shown in Table I, TBM was more prevalent in men, and the male to female ratio was 1.36:1 (109:80 patients). The mean duration of follow-up was $5 \pm 3.3$ months (range, 3-12 months). The number of patients in the current study with acute onset was $88(46.6 \%)$ cases, while subacute onset was observed in 67 (35.4\%) cases and chronic onset in $34(20.0 \%)$ cases. The average hospital stay of probable or possible TBM patients was $14.87 \pm 10.76$ days (95\% CI, 11.25-18.43 days). All patients presented with symptoms for $>1$ week, and the main complaints included fever $(78.3 \%)$, headache $(89.2 \%)$ and neurological complications (including focal neurological deficits, disturbance of consciousness and personality changes) in almost $40 \%$ of patients. In total, $6.3 \%$ of patients were in stage I, $69.3 \%$ were in stage II and $24.3 \%$ were in stage III (Table I). Furthermore, there were 34 patients with the increased intracranial pressure, who were treated by applying symptomatic and supportive treatment.

Laboratory data. The purified protein derivative (PPD) skin test was negative in 106 cases and positive in 83 cases. All patients underwent CSF cytology detection, and the CSF 


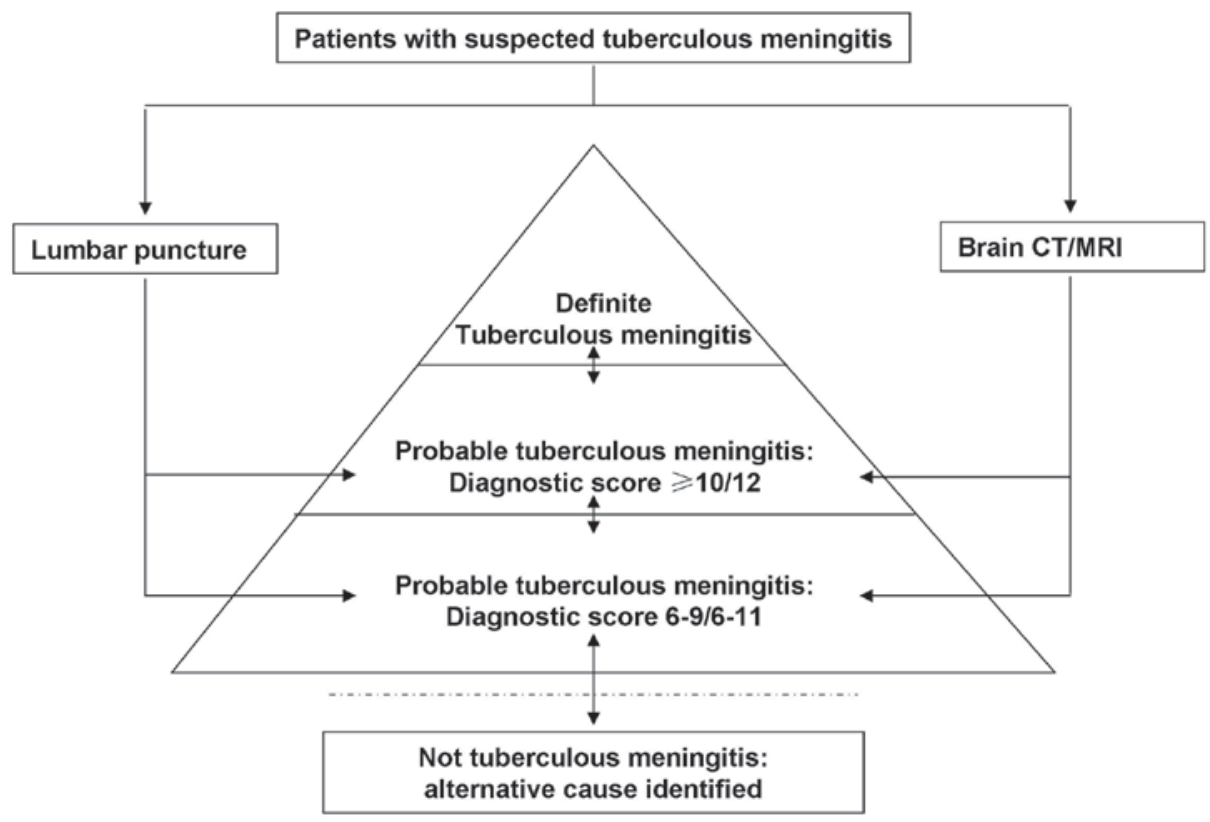

Figure 1. Categories of patients with suspected tuberculous meningitis. CT, computed tomography; MRI, magnetic resonance imaging.

findings on admission were as follows: A $13.9 \%$ of white blood cells and the quantity of CSF was $>500 / \mathrm{mm}^{3}$. Acid-fast bacilli (AFB) culture or smear detection was conducted in 173 cases, with only four cases $(2.3 \%)$ showing positive staining for Mycobacterium tuberculosis in an AFB smear and no positive cases in AFB culture test. In addition, 7 (18.9\%) cases presented positive results among 37 patients who underwent polymerase chain reaction detection of Mycobacterium tuberculosis DNA (Table II). Additionally, the TB antibody was examined in the CSF of 153 cases, with only 27 cases (17.6\%) demonstrating positive results.

Imaging data. In the imaging data of TBM patients, longer T1 and T2 signals were observed in the temporal lobe and thalamus (Fig. 2). According to the image, a large part of the brain nodules underwent meningeal enhancement. Furthermore, the cerebral ventricle and associated vessels were moderately expanded.

Treatment. Conventional 3HRZE/9HRZ treatment was administered to all the patients as described previously $(1,10)$, while symptomatic and supportive treatment was provided for reducing intracranial pressure, preventing arachnoid adhesions and reducing the CSF protein and sugar content. Patients with severe brain edema were administered glucocorticoid therapy. Following regular and rational anti-TB treatment, as well as supportive treatment, these patients achieved an improved clinical outcome, the improved clinical outcome rate is $87.3 \%$. In total, 7 cases presented hydrocephalus, and the fatality rate was only $1.58 \%(3 / 189)$.

Prognosis. The comprehensive treatment of the disease resulted in significant improvement in 145 cases, accounting for $76.7 \%$ of cases. In general, the cases were followed-up for 3-12 months and no recurrence was observed. There were 3 cases that succumbed to the disease with deteriorated, while 12 cases presented severe complications (6.5\%; including focal neurological deficits, disturbance of consciousness and personality changes) and 14 cases were discharged automatically or transferred for other reasons are unknown.

In addition, the fever reported in patients of the current retrospective study was mostly medium $\left(38-39^{\circ} \mathrm{C}\right)$ and high (40-41 $\mathrm{C})$, with only 43 cases $(29.1 \%)$ presenting peak fever temperature $\left(37-38^{\circ} \mathrm{C}\right)$.

\section{Discussion}

According to a published study, the number of annual incidence of TB in China is currently 100 million, accounting for $14.3 \%$ of the global TB incidence and ranking as the second highest incidence in the world (10). The results of the 'The fifth national tuberculosis epidemiological survey in 2010' (11) revealed that the prevalence of TB in China has declined very slowly. In the population aged $\geq 15$ years, the TB prevalence rate was reduced from $466 / 100,000$ in 2000 to $459 / 10$ million in 2010 (4). In China, the TB infection rate was $4.5 \%$ of the total population, and the incidence of TBM accounted for $\sim 1 \%$ of this rate.

TBM is a common infectious disease of the CNS, and results in high disability and mortality rates among severe infectious diseases. The main etiology of TBM is the infection with the TB bacterium, which is transported through the blood and directly infects the meninges, in which case TBM is a partial performance of the whole body blood line of disseminated TB. Another secondary consequence of TBM is CNS inflammation caused by invading Mycobacterium tuberculosis, which usually involves parts of body other than the lungs, including pleura, lymph nodes and genitourinary tract (10).

The clinical inflammation course of TBM can be divided into acute ( $\leq 14$ days), sub-acute (15-30 days) and chronic onset ( $\geq 31$ days). The clinical manifestations of TBM are non-characteristic, and common symptoms include headache $(50-80 \%)$, fever $(60-95 \%)$, loss of appetite and weight loss (60-80\%), and vomiting (30-60\%). Common signs are a stiff 
Table I. Clinical characteristics of probable or possible TBM cases.

\begin{tabular}{lr}
\hline Characteristics & $\mathrm{n}(\%)$ \\
\hline Sex & \\
Male & $109(57.7)$ \\
Female & $80(42.3)$ \\
Age range, years & \\
$\leq 18$ & $27(14.3)$ \\
18-35 & $67(35.4)$ \\
$35-65$ & $84(44.4)$ \\
$>65$ & $11(5.8)$ \\
Onset type & \\
Acute & $88(46.6)$ \\
Sub-acute & $67(35.4)$ \\
Chronic & $34(20.0)$ \\
Clinical stage & \\
Stage I & $12(6.3)$ \\
Stage II & $131(69.3)$ \\
Stage III & $46(24.3)$ \\
Symptoms & \\
Headache & $168(89.2)$ \\
Fever & $148(78.3)$ \\
Nausea/vomiting & $128(67.7)$ \\
Lack of appetite/weakness & $77(40.7)$ \\
Changes in personality & $71(37.6)$ \\
Weight loss & $52(27.5)$ \\
Night sweats & $47(24.9)$ \\
Stiff neck & $33(17.5)$ \\
Cranial nerve palsy & $29(15.3)$ \\
Blurred consciousness & \\
Convulsions & \\
Paraplegia/paresis & \\
\hline
\end{tabular}

neck (40-80\%), altered mental status (30-60\%), cranial nerve damage (30-50\%), mental abnormalities (10-30\%), hemiplegia (10-20\%), paraplegia (5-10\%) and seizures (5\% in adults; $50 \%$ in children) (12).

The retrospective analysis of suspected TBM patients conducted in the current study indicated that $82 \%$ of cases presented with acute and sub-acute onset. This high rate of diagnosis at the earlier stages of TBM may be associated with the gradual increase in the concern of the population over their health, as well as the increased awareness of TBM in recent decades. In addition, the Department of Neurology in the Xiangya Hospital of Central South University is the leading and professional institute for TBM treatment in China, and therefore may treat more patients with TBM symptoms (13). Patients with TBM often exhibit common symptoms, including fever, headache, cough and TB prior to the onset. It is widely considered that TBM patients commonly present low-grade fever $(2,10,11)$, however, the fever reported in patients of the current retrospective study was mostly medium and high, with only 43 cases $(29.1 \%)$ presenting peak fever temperature
Table II. Laboratory characteristics in the cerebrospinal fluid of tuberculous meningitis cases $(n=189)$.

\begin{tabular}{lr}
\hline Characteristics & $\mathrm{n}(\%)$ \\
\hline White blood cell count $/ \mathrm{mm}^{3}$ & \\
$\leq 100$ & $69(36.9)$ \\
$100-500$ & $92(49.2)$ \\
$\geq 500$ & $26(13.9)$ \\
Intracranial pressure $\left(\mathrm{mmH}_{2} \mathrm{O}\right)$ & \\
$\leq 180$ & $63(33.7)$ \\
$180-250$ & $78(41.7)$ \\
$\geq 250$ & $46(24.6)$ \\
Blood glucose level (mmol/l) & \\
$<0.60$ & $165(88.2)$ \\
$\leq 0.30$ & $117(62.6)$ \\
Protein level (g/l) & \\
$\leq 0.45$ & $21(11.2)$ \\
$0.45-2$ & $122(65.2)$ \\
$\geq 2$ & $44(23.5)$ \\
Acid-fast bacilli culture Mycobacterium & $4(2.3)$ \\
tuberculosis positivity (n=173) & \\
\end{tabular}

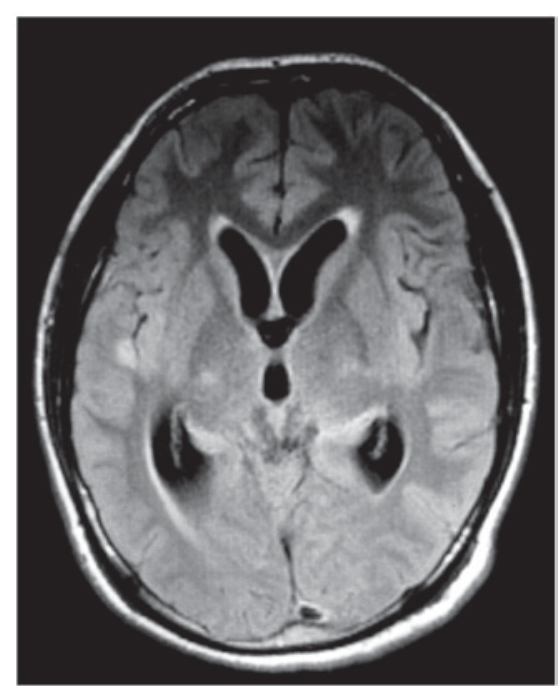

Figure 2. Imaging data of a representative tuberculous meningitis patient. In the right temporal lobe and thalamus, the strengthened $\mathrm{T} 1, \mathrm{~T} 2$ signals could be observed, which indicated that a large part of the brain nodules underwent meningeal enhancement. Furthermore, the cerebral ventricle and associated vessels were moderately expanded.

$\left(37-38^{\circ} \mathrm{C}\right)$, which is not consistent with previous studies. This inconsistency in observations may be associated with the mutation of Mycobacterium tuberculosis and the high reproduction of Rich foci. In the present study, 138 (73.0\%) cases had stiff neck, while clinical fever was reported in 148 cases $(78.3 \%)$, headache in 168 cases $(89.2 \%)$ and vomiting in 128 patients (67.7\%) (Table I). Therefore, in order to prevent misdiagnosis, clinical symptoms (including headache and fever) should be taken into account during TBM diagnosis.

TBM diagnosis is based primarily on CSF smear detection or CSF culture of isolated Mycobacterium tuberculosis. 
However, the positive rate of detection for the smear and culture tests is low (14). CSF smears for acid-fast staining etiological diagnosis is the most efficient method compared with the traditional methods, however, the smear microscopy for AFB-positive rate in the present study was $2.3 \%$, which was lower than the rates of 37 to $87 \%$ reported in the other studies $(9,11,14)$. Thwaites et al $(15)$ conducted Ziehl-Neelsen staining in CSF samples from 132 TBM patients and reported 77 cases of AFB detection, with a detection rate of up to $58 \%$. The study observed that the detection of a large quantity of CSF ( $>6 \mathrm{ml}$ ) was a direct method to discover the AFB smear independent factors, while an average of 30-min microscopy time and repeated testing helps to improve the detection rate (15). In addition, the length of the TBM course, CSF and neutrophils ratio, CSF lactate content, and CSF and plasma glucose ratio are associated with the AFB detection rate (15). Furthermore, a previous meta-analysis indicated that nucleic acid amplification testing in the diagnosis of TBM had a sensitivity and specificity of 56 and $98 \%$, respectively (16). The small amount of CSF samples in the present study may be the main reason for the low rate of positive AFB observed.

It has been reported that $>2 / 3$ of TBM patients have increased CSF pressure (220-500 $\left.\mathrm{mm} \mathrm{H}_{2} \mathrm{O}\right)$, therefore, it's critical for the TBM diagnosis to exclude the other factors that affect the CSF pressure, including mental stress, suffocation, coughing, and increased chest and abdominal cavity pressure. In addition, the intracranial pressure is always low in the presence of sub-arachnoid adhesions or obstruction (16). In the present study, $66.3 \%$ of patients exhibited increased intracranial pressure $\left(>180 \mathrm{~mm} \mathrm{H}_{2} \mathrm{O}\right)$, however, a previous study demonstrated $\sim 7.5 \%$ patients with intracranial pressure of $>100 \mathrm{~mm} \mathrm{H}_{2} \mathrm{O}$ (11). Total of 59 patients with lower intracranial pressure than normal, and even 4 patients' pressure could not be tested which considered with varying degrees of high obstruction. It should be noted the intracranial tuberculoma or abscess oppression, venous sinus thrombosis and subarachnoid hemorrhage condition are observed when intracranial pressure is high $\left(>400 \mathrm{~mm} \mathrm{H}_{2} \mathrm{O}\right)$. If the above higher intracranial pressures $\left(>400 \mathrm{~mm} \mathrm{H}_{2} \mathrm{O}\right)$ occurred, the doctor closely monitor these patients. The CSF in patients with TBM in the acute phase commonly exhibit an increased number of neutrophils, which are converted into hybrid cells (derived from stem cells that have an important role in organogenesis, tissue regeneration and cancer formation) in the subacute phase, and an increased number of lymphocytes is detected in the tissue repair stage $(15,16)$. CSF cytological studies $(15,16)$ have demonstrated that the increased neutrophils in $20-40 \%$ patients were usually changed to lymphocyte dominance in the subsequent $24-48 \mathrm{~h}$. In the present study, this neutrophilic predominance was present in $16.4 \%$ of patients on admission and changed into lymphocytic predominance within a few days. However, all CSF cytology tests demonstrated a few interference factors, and doctors should pay more attention to distinguish from the purulent meningitis in the clinic.

The CSF protein levels in TBM patients increased in 165 out of 189 cases $(87.3 \%)$ in the present study, with $\geq 5 \mathrm{~g} / \mathrm{l}$ in 5 patients $(2.6 \%)$ considered to be due to spinal canal obstruction. Compared with cryptococcal meningitis (15), CSF protein content in TBM was increased to a greater extent, and protein levels of $>2 \mathrm{~g} / \mathrm{l}$ suggested a greater possibility of TBM. In addition, another important basis for the diagnosis of TBM was the fact that the CSF glucose generally exhibited a moderate reduction. There were 153 out of the 189 patients (80.9\%) who presented decreased glucose level, which was similar to the findings of previous studies $(10,16)$. Although the retrospective analysis of glucose content demonstrated reduction in 92 patients (48.7\%), as blood sugar and ion factors were not excluded, the absolute glucose content of CSF in TBM in the present study was not fully determined. However, the reasons for not excluding blood sugar and ion factor in the present study included: The patients' condition was mild or in the early stage, the patient exhibited alternative underlying diseases and their respective long-term drug treatments affected the laboratory markers, in intravenous saline or glucose levels. However, in the present study, the atypical cases of CSF caused due to the variability of Mycobacterium tuberculosis were significantly increased. Consequently, too much dependence on testing cell count, glucose and chloride changes for diagnosing TBM typically results in misdiagnosis of TBM $(16,17)$. Furthermore, as demonstrated by the statistical results of the present study, the positive rates of patients with TBM for PPD skin test and PPD antibody tests were lower compared with the number of negative rates, which may be due to the fact that the study patients were adults, and these tests may have a higher positive rate in children.

The performance of head computed tomography (CT) and magnetic resonance imaging (MRI) in TBM patients is relatively similar, although MRI is superior to CT, with the most important change reflected in the asymmetric cisterna ambiens, Sylvian cistern and the abnormal anatomy region on the brain (18). The degree of enhancement during MRI cisternography is superior to that in CT, which may be associated with meningeal infiltration (18). MRI scanning can clearly demonstrate early-stage or small lesions, as well as reflect the size and shape of the lesions and different tissue components of the lesion, whereas $\mathrm{CT}$ is better at displaying calcification (18). It has been reported that positive rate of TBM in CT examination is as high as $80 \%$, while the rate in MRI scans is even higher; thus, performing CT or MRI examination is essential for the diagnosis of TBM $(11,15,16)$. In the current study, 144 patients underwent head imaging studies, of which 127 cases $(88.3 \%)$ presented abnormal findings. The positive detection rate in the present study was lower compared with that reported in other studies $(14,15)$, and this discrepancy may be due to the following main reasons: i) Patients were admitted at the early onset of the disease and alterations in the brain had not yet developed; ii) patients did not undergo head MRI or CT enhancement examination due to economic or other problems; and iii) the reviewer did not have a clear understanding to the TBM imaging changes. In the present study, it is recommended that dynamically imaging examination should be performed for TBM diagnosis. Imaging also identified patients with hydrocephalus in 7 cases $(4.86 \%)$, as observed by retrospective analysis. Previous research has demonstrated that brain effusion serves a certain role in TBM prognosis, commonly resulting in poorer patient prognosis (18). Long-term brain effusion leads to increased intracranial pressure, which oppresses the nerve in the skull base and brain parenchyma, resulting in facial paralysis, blindness, strabismus, mental retardation, aphasia, paralysis or other consequences $(11,16)$. 
Thus, early detection and appropriate management of brain effusion can improve the prognosis.

Clinically, the treatment of TBM usually involves anti-TB therapy supplemented by symptomatic and supportive treatment. Anti-TB treatment is required to comply with the principle of early supervision, combined supervision, regular supervision, adequate supervision and the full supervision (11). During treatment, a particular drug that is able to go through the blood-brain barrier and reach higher blood concentration in the CSF should be selected for TBM. For the initial treatment of patients with TBM mainly includes the HREP and O/HRESO programs in China, consistent with the strategies reported in other countries $(1,11)$. If the patient develops resistance, then second-line anti-TB drugs are used, such as isoniazid aminosalicylate tablets and prothionamide plus amikacin therapy $(9,15)$.

During hospital admission in the present study, $6.3 \%$ of the cases were stage I, $69.3 \%$ were stage II and $24.3 \%$ were stage III (Table I). At this time, $37.6 \%$ of the patients presented altered mental status, $17.5 \%$ had convulsions, $48.1 \%$ presented blurred consciousness, and $15.3 \%$ presented paresis/paraplegia. In addition, $23 \%$ of patients suffered from an underlying disease, including gastritis, hepatitis, diabetes, coronary heart disease, trauma, malignancy and alcoholism. Particularly, factors such as diabetes, malignancy and alcoholism are known to serve a role in TBM development; therefore, they may also be considered to contribute to a poor prognosis (19). However, the mortality rate in the present study was $1.58 \%$, which is lower in comparison with other studies in China and abroad $(11,15,16)$. This may be associated with the following factors: i) The disease duration of patients in the current study was mostly in the early or middle period, and the symptoms were mild; ii) the patient cohort included mostly young men, whose immune system was normal, with only a few cases with human immunodeficiency virus infection or other autoimmune diseases; and finally; and iii) once TBM was considered, the patients were administered anti-TB therapy along with symptomatic and supportive treatment. Furthermore, it should be noted that certain patients that survived requested discharge due to economic or other reasons.

Although the present study identified various significant findings, there were also certain limitations. Firstly, the most significant limitation is the TB-spot test, which was not performed in all the patients. The TB-spot test is widely used worldwide, and thus this test should be used for TB infection diagnosis in future studies. Furthermore, the current study did not include cases with other meningitis types, caused by the bacteria or a virus. The present study is only a preliminary analysis of TBM in Chinese patients, and in following studies, cases that involved other meningitis types caused by bacteria will be used as the control group, in order to clarify the diagnosis of TBM.

In the present study, 189 patients with confirmed or presumed TBM reported over a 6-year period were analyzed retrospectively. The present study examined the clinical, laboratory and neuroradiological findings of patients with TBM, and evaluated the prognostic significance of these variables. In addition, the local epidemiological data were reported from these cases. In conclusion, the pathogenesis and clinical manifestations of TBM were variable. In patients with TBM, the typical alterations of CSF and brain imaging are rarely identified, therefore, the diagnosis for TBM must be made by combining these with with the findings of laboratory tests, which may subsequently reduce the misdiagnosis rate. Thus, the diagnosis of infection of the CNS should be considered along with the clinical manifestations, CSF examination and treatment effect. Comprehensively investigating clinical manifestations, carefully reviewing radiology data and examining microbiological and pathological evidence of TB are extremely important for establishing the correct diagnosis. In certain cases, a therapeutic trial of anti-TB therapy is required in order to avoid delaying the treatment and adversely affecting the patient's condition. Furthermore, the present data combined with the British Infection Society guidelines (12) and the standardized clinical case definition for TB, maybe used to develop effective and applicable TBM treatment.

\section{Acknowledgements}

Not applicable.

\section{Funding}

The present study was funded by a grant from the Fundamental Research Funds for Central Universities of Central South University (grant no. 2011ssxt213).

\section{Availability of data and materials}

All data generated or analyzed during this study are included in this published article.

\section{Authors' contributions}

ML, WW, QZ and XY analyzed and interpreted the patient data. ML, YL, HY performed the experiments. ML and XY were the major contributors in writing the manuscript. All authors read and approved the final manuscript.

\section{Ethics approval and consent to participate}

All of the patients provided their prior informed written consent for the use of their samples in the present study.

\section{Consent for publication}

All of the patients provided their prior written informed consent for the present study.

\section{Competing interests}

The author declared no conflict of interests.

\section{References}

1. Chin H: Tuberculous meningitis: Diagnostic and therapeutic challenges. Neurol Clin Pract 4: 199-205, 2014.

2. Bartzatt R: Tuberculosis infections of the central nervous system. Cent Nerv Syst Agents Med Chem 11: 321-327, 2011.

3. World Health Organization: Global Tuberculosis Report 2013. http://www.who.int/tb/publications/global_report/en/index.html. Accessed January 10, 2014. 
4. Christense AS, Andersen AB, Thomsen VO, Andersen PH and Johansen JS: Tuberculous meningitis in Denmark: A review of 50 cases. BMC Infect Dis 11: 47, 2011.

5. Yuchong C, Fubin C, Jianghan C, Fenglian W, Nan X, Minghui Y, Yalin S and Zhizhong Z: Cryptoccosis in China (1985-2010): Review of cases from Chinese population. Mycopathologia 173: 329-335, 2012

6. Qu J, Zhou T, Zhong C, Deng R and Lü X: Comparison of clinical features and prognostic factors in HIV-negative adults with cryptococcal meningitis and tuberculous meningitis: A retrospective study. BMC Infect Dis 17: 51, 2017.

7. World Health Organization: Treatment of tuberculosis guidelines. 4th edition. http://whqlibdoc.who.int/publications/2010/ 9789241547833_eng.pdf.

8. Prasad K and Singh MB: Corticosteroids for managing tuberculous meningitis. Cochrane Database Syst Rev: CD002244, 2008.

9. Marais S, Thwaites G, Schoeman JF, Török ME, Misra UK, Prasad K, Donald PR, Wilkinson RJ and Marais BJ: Tuberculous meningitis: A uniform case definition for use in clinical research. Lancet Infect Dis 10: 803-812, 2010.

10. Lopez-Varela E and García-Basteiro AL: World health organization guidenlines for children tuberculosis management: Successes achieved and challenges ahead. Pediatr Infect Dis J 33: 1310-1311, 2014.

11. Techinical Guidance Group of the Fifth National TB Epi-demiological Survery and the Office of the Fifth National TB Epidemiological Survey: The Fifth National Tuberculosis Epidemiological Survey in 2010. Chin J Antituberculosis 34 485-508, 2012.
12. Thwaites G, Fisher M, Hemingway C, Scott G, Solomon T and Innes J; British Infection Society: British Infection Society guidelines for the diagnosis and treatment of tuberculosis of the central nervous system in adults and children. J Infect 59: 167-187, 2009.

13. Zhang $\mathrm{W}, \mathrm{Hu} \mathrm{Z}$ and $\mathrm{Li} \mathrm{T}$ : A case of tuberculous meningitis with atypical multiple lesions. West Indian Med J 63: 789-790, 2014.

14. Mathai A, Radhakrishnan VV, George SM and Sarada C: A newer approach for the laboratory diagnosis of tuberculous meningitis. Diagn Microbiol Infect Dis 39: 225-228, 2001.

15. Thwaites GE, Chau TT and Farrar JJ: Improving the bacteriological diagnosis of tuberculous meningitis. J Clin Microbiol 42: 378-379, 2004

16. Pai M,Flores LL, Pai N, Hubbard A, Riley LW and Colford JM Jr: Diagnostic accuracy of nucleic acid amplification tests for tuberculous meningitis: A systematic review and meta-analysis. Lancet Infect Dis 3: 633-643, 2003.

17. Thwaites GE and Tran TH: Tuberculous meningitis: Many questions, too few answers. Lancet Neurol 4: 160-170, 2005.

18. Kalita J, Misra UK and Ranjan P: Tuberculous meningitis with pulmonary miliary tuberculosis: A clinicoradiological study. Neurol India 52: 194-196, 2004.

19. Garg RK, Malhotra HS and Gupta R: Spinal cord involvement in tuberculous meningitis. Spinal Cord 53: 649-657, 2015. 